\title{
Neo Adjuvant Chemotherapy in Operable Breast Cancer - A Single Centre Study
}

\author{
Chandan M. N. ${ }^{1}$, Bhuyan K. ${ }^{2}$ \\ ${ }^{1}$ Department of Surgical Oncology, Gauhati Medical College and Hospital, Guwahati, Assam, India. \\ ${ }^{2}$ Department of General Surgery, Gauhati Medical College and Hospital, Guwahati, Assam, India.
}

\section{ABSTRACT}

\section{BACKGROUND}

Breast cancer is the most common cancer in females worldwide. Management of breast cancer has changed over the past several years from radical to more of conservative approaches. The present study was undertaken to evaluate the role of neoadjuvant chemotherapy in downsizing large operable breast cancer and feasibility of less radical, breast conservative surgery (BCS).

\section{METHODS}

A prospective study of 42 patients with operable breast cancer was carried out over a period of one year. Patients with stage IIIA disease of 18-50 years of age were included. Clinical tumour response to NACT was graded according to the World Health Organization Criteria for clinical tumour response. Loco-regional management consisted of either BCS or Modified Radical Mastectomy (MRM), depending on feasibility and patients' choice of surgery.

\section{RESULTS}

The mean age of patients was 43.73 years. Most of the patients were premenopausal. Statistically significant response was achieved in downsizing the tumour with complete clinical response (cCR) in $12.5 \%$, and partial clinical response (CPR) in 75\% patients. BCS became feasible for $68.75 \%$ patients post neoadjuvant chemotherapy.

\section{CONCLUSIONS}

Neoadjuvant Systemic Therapy is effective in the management of operable breast cancer. BCS was feasible in majority of cases.
Corresponding Author:

Dr. Bhuyan K.,

Professor,

Department of General Surgery, GMCH, Guwahati-781032, Assam, India

E-mail: kanakbhuyan@gmail.com

DOI: $10.14260 /$ jemds/2019/859

Financial or Other Competing Interests: None.

How to Cite This Article:

Chandan MN, Bhuyan K. Neo adjuvant chemotherapy in operable breast cancer- a single centre study. J. Evolution Med. Dent. Sci. 2019;8(52):3972-3976, DOI: 10. 14260/jemds/2019/859

Submission 03-11-2019, Peer Review 11-12-2019,

Acceptance 18-12-2019,

Published 30-12-2019.

\section{KEY WORDS}

Ca Breast, Neo-Adjuvant, BCS, MRM 


\section{BACKGROUND}

Breast cancer is the most common cancer worldwide representing nearly $25 \%$ of all cancer cases and accounting for $15 \%$ of all cancer deaths. (1) Breast cancer is the most common cancer among Indian females as well with age adjusted incidence rate of 25.8 per 100,000 women with mortality of 12.7 per 100,000 women. (2) The high mortality may be due to lack of adequate breast cancer screening and late diagnosis due to lack of awareness. Standard prognostic indicators such as number of involved lymph nodes, size of tumour are not applicable in the neoadjuvant setting. Identification of prognostic variables for patients receiving neoadjuvant chemotherapy becomes most important to breast cancer multi-disciplinary management decisions. The pathological response to neoadjuvant chemotherapy may be an outcome indicator in neoadjuvant setting. Even though surgery is the mainstay in the management of carcinoma breast, chemotherapy has been currently delivered in the preoperative (Neoadjuvant) setting prior to definitive surgery and is now considered a widely accepted option. (3) It is postulated that neo-adjuvant chemotherapy may diminish the potential of micro metastases in breast cancer patients with enhanced survival. $(4,5)$

Early diagnosis and early systemic treatment of breast cancer with neo-adjuvant chemotherapy provides better results. The aim of the study was to analyse the response to neo-adjuvant chemotherapy in patients with the objective to downsize the tumour prior to definitive surgical intervention.

\section{METHODS}

In this Prospective Interventional study of consecutive 42 cases of diagnosed breast cancer were taken up for the study. Patients with early breast carcinoma, advanced breast carcinoma were included in the study. Institutional Ethics committee approved the study.

As per the triple assessment the patients were analysed clinically, radiologically and pathologically. Thorough examination of both the breasts, axillae and all the systems were done and noted before the start of $1^{\text {st }}$ cycle NACT and after completion of NACT. Tumours were measured bidimensionally (longest perpendicular diameters in $\mathrm{cm}$ ). Patients were staged clinically using TNM staging for further management. Histopathological examination (HPE) along with assessment of hormone receptor status. On the basis of HPE report patients were categorized into 2 groups: in situ breast carcinoma and invasive breast carcinoma. DCIS and LCIS came under in situ breast carcinoma. Invasive breast cancer further divided into invasive ductal carcinoma (IDC) and invasive lobular carcinoma (ILC). Based on hormonal status patients were divided into two groups for NACT: HER 2 Neu negative group and the other was HER 2 Neu positive $(3+)$ group.

According to NCCN guidelines version 1. 2017 (NCCN. org/patients) HER 2 Neu negative patients received 4 cycles of docetaxel, doxorubicin/adriamycin and cyclophosphamide (DAC) every three weeks. HER 2 Neu positive patients received 4 cycles of dose dense doxorubin/adriamycin and cyclophosphamide and 4 cycles of paclitaxel and trastuzumab every two weeks with standard doses determined by body surface area (BSA). After 4 cycles of chemotherapy patients were examined for response. The clinical response of dimensionally measurable and assessable disease was classified as complete response (cCR), partial response (cPR), stable disease (cSD), or progressive disease (CPD) according to WHO criteria. cCR was defined as the disappearance of all clinical evidence of tumour; cPR was defined as a $50 \%$ or more reduction in the sum of the products of measured lesions, or estimated decrease in tumour size of at least $50 \%$, without the appearance of new lesions; cSD was defined as a decrease in lesion size of less than $50 \%$ in the sum of the products of measured lesions and/or increase of less than $25 \%$, without the appearance of new lesions. cPD is defined as any measured or estimated increase greater than $25 \%$ or the appearance of new lesions.

After assessing tumour response in breast cancer patients' definitive surgery was planned following completion of NACT. Definitive Surgery performed after 3 weeks of completion of NACT with either of MRM or BCT. Pathological response was assessed from histopathological examination of the specimen. pCR (Pathological complete response) is defined as the absence of residual invasive and in situ cancer in resected breast specimen and all sampled regional lymph nodes. pPR (Pathological partial response) was defined as presence of invasive carcinoma with stromal alterations in resected breast specimen and all sampled regional lymph nodes. pNR (Pathological no response) was defined as little modification in the original tumour appearance in complete resected breast specimen and all sampled regional lymph nodes.

Statistical Analysis: Baseline demographic characteristics and microscopic findings were compiled. Response to neoadjuvant chemotherapy was also found out. Possible risk factors affecting tumour response and lymph node response were tested using tests of proportions (Pearson's chi square test and Fischer's exact test whichever was applicable) and $p$ value was calculated. Relative risk (RR) and 95\% confidence interval (CI) were also calculated from the data. All the data were analysed with the software GraphPad InStat 3 for windows.

\section{RESULTS}

In this study, majority of the patients were of the age group between 40 and 50 years. 24 patients under 50 years' age group (57. $15 \%$ ) and 18 patients above 50 years of age ( 42 . $85 \%), 39$ (92. 86\%) and 3 were unmarried with 7. 14\% (Table 1). Mean tumour size in the present study was 5. 87 cm with standard deviation of 1. 2. 7 of them had T2 status (16. $67 \%$ ) i. e., tumour size $<5 \mathrm{~cm}$ and 21 of them had T3 status (50\%). 14 of them had T4 status (33.33\%. 6 of them had N0 status with $14.29 \%$ and 27 of them had N1 status with $64.29 \%$ and 9 of them had N2 status with $21.42 \%$.

34 of them diagnosed with Invasive ductal carcinoma (IDC) with $80.95 \%$ and 1 of them was Invasive lobular carcinoma (ILC) with $2.4 \% .7$ of them had ductal carcinoma in situ with 16. 67\% (Table 4). Immunohistochemistry (Hormone receptor status) of 42 female patients with carcinoma breast are as observed, 32 of them are Oestrogen 
Receptor (ER) positive with 76. 19\% and 10 of them were ER negative with $23.81 \%$. 26 of them were Progesterone receptor positive with $61.19 \%$ and 16 of them were progesterone receptor negative with 38.09\%. 10 patients were HER 2 Neu positive with $23.81 \%$ and 32 were negative with 76. 19\%. 6 triple positive with 14. $29 \%$ and 6 triple negative with 14. 29\% (Table 2). As per NCCN guidelines, HER $2 \mathrm{Neu}+\mathrm{ve}$ received dose dense chemotherapy i. e., adriamycin and cyclophosphamide with paclitaxel and trastuzumab (AC\&PT) whereas HER $2 \mathrm{Neu}$-ve received docetaxel, adriamycin and cyclophosphamide (DAC). Out of 42 patients, 32 of them (76.19\%) received DAC regimen and $10(23.81 \%)$ of them received AC \& PT regimen. Following NACT, 10 patients showed complete clinical response (cCR) with 23. 8\%, 28 patients showed partial clinical response (cPR) with $66.67 \%$ and 4 patients had clinically stable disease (cSD) with $9.52 \%$ and none of the patient showed (cPD) clinically progressive disease.

Following completion of NACT, 38 (90.48\%) patients underwent MRM and 4 of them underwent BCS (9. 52\%). In this study, pathological complete response between two age groups $\leq 50$ years and $>50$ years were assessed after NACT and it was found that 4 cases were of the age group $\leq 50$ years $(16.67 \%)$ and 2 cases were $>50$ years of age $(11.11 \%)$. Pathological partial response to NACT was found in20 cases in the age group $\leq 50$ years (83. 33\%) and 16 cases were of $>50$ yrs., of age (88. 89\%) (Table 3). In this study, majority of the cases presented with axillary node involvement with 36 cases $(86 \%)$ which corresponds to N1 \& N2 nodal status and without axillary node involvement is 6 cases (14\%) corresponding to N0 nodal status. 3 cases (50\%) with axillary node involvement showed complete pathological response and 3 cases $(50 \%)$ showed partial pathological response to NACT. 3 cases (8. 33\%) without axillary node involvement showed complete pathological response and 33 cases $(91$. $7 \%$ ) showed partial pathological response to NACT with $95 \%$ confidence interval of 1.5 and RR of 6 and p value of 0.0291 which is statistically significant. In this study, majority of the cases diagnosed with invasive ductal carcinoma with 34 cases (table 4) and invasive lobular carcinoma with 1 case and 7 cases of non-invasive DCIS. 3 cases (8. 6\%) with invasive histological features showed complete pathological response and 32 cases (91. 4\%) showed partial pathological response. 3 cases (42. 86\%) with non-invasive histological features showed complete pathological response and 4 cases (57. $14 \%$ ) showed partial pathological response with $95 \%$ confidence interval of 0.050 and RR of 0.2 and p value of 0 . 0458 which is statistically significant.

In this study, majority of the cases had ER receptor positive status with 32 cases and ER negative status of 10 cases. 2 cases (6. 25\%) with ER receptor positive status showed complete pathological response and 30 cases $(93$. $75 \%$ ) showed partial pathological response to NACT. 4 cases (40\%) with ER negative status showed complete pathological response and 6 cases (60\%) showed partial pathological response to NACT with $95 \%$ confidence interval of $0.03, \mathrm{RR}$ of 0.156 and $\mathrm{p}$ value of 0.0214 which is statistically significant. In this study, majority of the cases had PR positive status with 26 cases and PR negative status of 16 cases. 1 case (4\%) with PR receptor positive status showed complete pathological response and 24 cases (96\%) showed partial pathological response to NACT. 5 cases (29. 4\%) with PR negative status showed complete pathological response and 12 cases (70.6\%) showed partial pathological response to NACT which lie in 95\% confidence interval with RR of 0.136 and $\mathrm{p}$ value 0.0318 which is statistically significant (Figure 1).

In this study, majority of the cases had HER 2 Neu negative status with 32 cases (Figure 2) and HER 2 Neu positive status of 10 cases. 4 cases (40\%) with HER 2 Neu positive status showed complete pathological response and 6 cases $(60 \%)$ showed partial pathological response to NACT. 2 cases (6. 25\%) with HER 2 Neu negative status showed complete pathological response and 30 cases (93. 75\%) showed partial pathological response to NACT with RR of 6.4 and $p$ value of 0.0214 which is statistically significant. In post-operative specimen 6 of the tumours showed complete pathological response (pCR) with 14. $29 \%$ and 36 of them showed partial pathological response (pPR) with $85.71 \%$.

\begin{tabular}{|c|c|c|c|}
\hline \multicolumn{2}{|c|}{ Characteristics } & Number & Percentage \\
\cline { 2 - 4 } Age & $\leq 50 \mathrm{Y}$ & 24 & 57.15 \\
\hline \multirow{2}{*}{ Sex } & $>50 \mathrm{Y}$ & 18 & 42.85 \\
\cline { 2 - 4 } & Female & 42 & 100 \\
\cline { 2 - 4 } & Male & 00 & 0 \\
\hline \multirow{2}{*}{ Marital status } & Married & 39 & 92.86 \\
\cline { 2 - 4 } & Unmarried & 3 & 7.14 \\
\hline \multirow{2}{*}{ Menstrual status } & Premenopause & 27 & 64.23 \\
\cline { 2 - 4 } & Post menopause & 15 & 35.77 \\
\hline \multirow{2}{*}{ Exclusive breast feeding } & Yes & 39 & 92.86 \\
\cline { 2 - 4 } & No & 7.14 \\
\hline \multicolumn{2}{|c|}{ Table 1. Demographic Characteristics of Breast Cancer Patients } \\
\hline
\end{tabular}

\begin{tabular}{|c|c|c|}
\hline Hormone Receptor Status & Number & Percentage \\
\hline ER +ve & 32 & $76.19 \%$ \\
\hline ER -ve & 10 & $23.81 \%$ \\
\hline PR +ve & 26 & $61.91 \%$ \\
\hline PR -ve & 16 & $38.09 \%$ \\
\hline HER 2 Neu +ve & 10 & $23.81 \%$ \\
\hline HER 2 Neu -ve & 32 & $76.19 \%$ \\
\hline Triple +ve & 6 & $14.29 \%$ \\
\hline Triple -ve & 6 & $14.29 \%$ \\
\hline Table 2. Hormone Receptor Status of Breast Cancer Patients \\
\hline
\end{tabular}

\begin{tabular}{|c|c|c|}
\hline \multirow{2}{*}{ Age Group (Y) } & Pathological Response \\
\cline { 2 - 3 } & Complete Response & Partial Response \\
\hline$\leq 50$ & $4(16.67 \%)$ & $20(83.33 \%)$ \\
\hline$>50$ & $2(11.11 \%)$ & $16(88.89 \%)$ \\
\hline \multicolumn{2}{|c|}{ Table 3. Pathological Response of Breast Cancer Patients to NACT in } \\
Different Age Groups \\
\hline
\end{tabular}

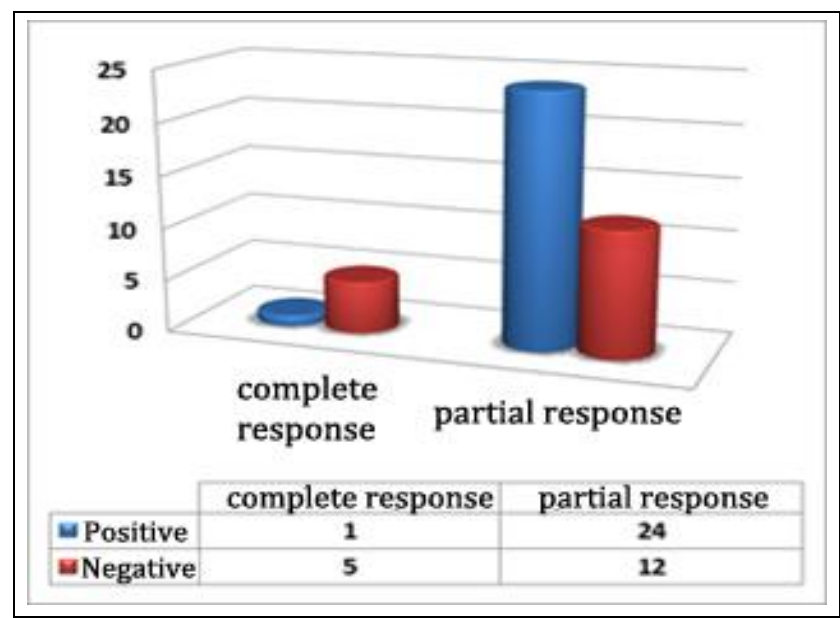

Figure 1. Pathological Response of Breast Cancer Patients to NACT According to PR Status 


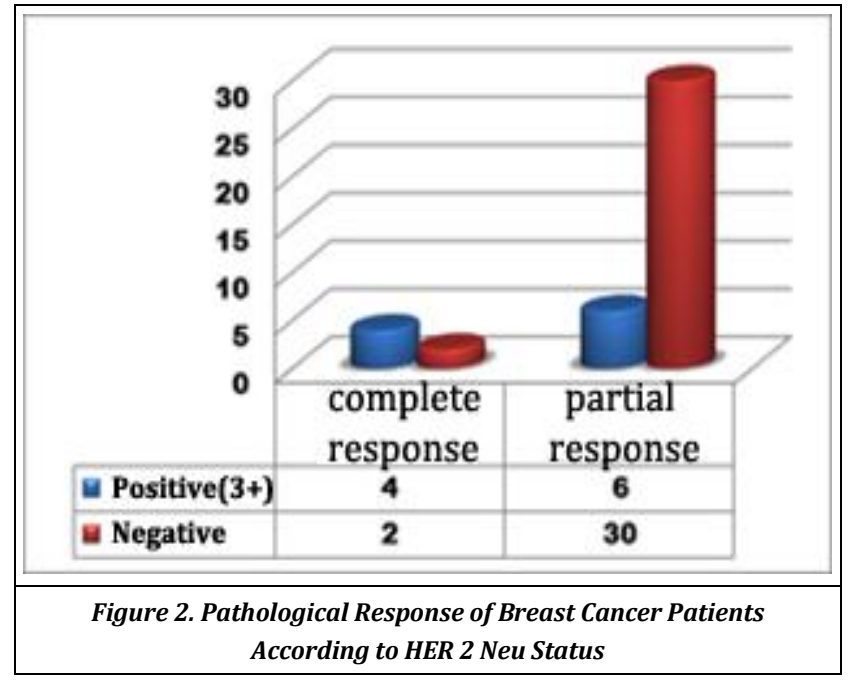

\section{DISCUSSION}

Neoadjuvant chemotherapy (NACT) is extensively used in the management in breast cancer. A recent publication from the National Cancer Institute Conference Statement on NACT suggests that initial TNM stage at presentation and response to NACT should be utilized to guide further loco-regional and possibly systemic therapy. (6) The Mean age of breast cancer patients in this study was 49 . 91 years with a standard deviation of 6.924 which is in accordance with studies. $(7,8)$ Mean tumour size of breast cancer patients at presentation was $5.87 \mathrm{~cm}$ with a standard deviation of 1.201 which is in accordance with other studies. $(9,10)$ In this study, majority of the cases (34: 80. 95\%) was diagnosed with invasive ductal carcinoma. In a published series the most common type of breast carcinoma was those of invasive duct carcinoma which comprised of $40-75 \%$. (11) In other series invasive duct carcinoma was found in $88 \%$ and $79.5 \%(7,12)$ which were in accordance with present study. Majority of the breast cancer patients in this study were $<50$ years of age with $57.15 \%$ $(\mathrm{n}=27)$ patients. Pathological Complete response (pCR) and pathological partial response (pPR) in this group were 16 $67 \%$ and $83.33 \%$ respectively. This is in accordance with other studies. (9) The Pathological response in $>50$ age groups showed pCR and pPR as $11.11 \%$ and $88.89 \%$ respectively. This is in accordance with a meta-analysis. $(9,13)$ Age group $<50$ years show better results for pCR to NACT.

In this study most of the patients presented with tumour size $>5 \mathrm{cms}$ i. e., 35 (83\%) patients. Pathological complete response $(\mathrm{pCR})$ and pathological partial response (pPR) in pre NACT tumour size $>5 \mathrm{cms}$ are $8.6 \%$ and $91.4 \%$ respectively. These are in accordance with other studies. $(8,9$, 13) The pCR and pPR in pre NACT breast cancer patients with tumour size $<5 \mathrm{cms}$ were of $42.86 \%$ and $57.14 \%$ respectively. The difference between the groups in this present study is statistically significant $(\mathrm{P}=0.0478)$. Patients with tumour size $<5 \mathrm{cms}$ at presentation show better response for pCR following NACT. In this study $86 \%$ of breast cancer patients $(n=36)$ presented with axillary node involvement. Pathological complete response (pCR) and pathological partial response (pPR) in axillary node involvement are $8.33 \%$ and $91.7 \%$ respectively. This is in accordance with other study. (9) The pCR and pPR without axillary node involvement to NACT are $50 \%$. This is in accordance with other study. (9) The difference between the two groups in this present study with respect of axillary node status in response to NACT is statistically significant $(p=0$. 0291).

\section{CONCLUSIONS}

Neo-adjuvant chemotherapy could be the standard of management in Stage II disease. Hormone-receptor negative and HER 2 Neu positive patients showed better response. Histopathological examination of the specimen following definitive surgery could be the best way of assessing the tumour response. Pathological complete response can be used as a surrogate biomarker to assess the tumour response to NACT.

\section{REFERENCES}

[1] Torre LA, Bray F, Siegel RL, et al. Global cancer statistics, 2012. CA: A Cancer Journal for Clinicians 2015;65 (2) :87-108.

[2] De Lena M, Zucali R, Viganotti G, et al. Combined chemotherapy-radiotherapy approach in locally advanced ( $\mathrm{T}$ 3b- $\mathrm{T}$ 4) breast cancer. Cancer Chemotherapy and Pharmacology 1978;1 (1) :53-9.

[3] Fisher B, Saffer E, Rudock C, et al. Effect of local or systemic treatment prior to primary tumour removal on the production and response to a serum growthstimulating factor in mice. Cancer Res 1989;49 (8) :2002-4.

[4] Bear HD, Anderson S, Smith RE, et al. Sequential preoperative or postoperative docetaxel added to preoperative doxorubicin plus cyclophosphamide for operable breast cancer: National Surgical Adjuvant Breast and Bowel Project Protocol B-27. J Clin Oncol 2006;24 (13) :2019-27.

[5] Buchholz TA, Lehman CD, Harris JR, et al. Statement of the scienceconcerning locoregional treatments after preoperative chemotherapy for breast cancer: a National Cancer Institute conference. J Clin Oncol 2008;26 (5) :791-7.

[6] Chang HR, Glaspy J, Allison MA, et al. Differential response of triple negative breast cancer to a docetaxel and carboplatin based neoadjuvant treatment. Cancer 2010;116 (18):4227-37.

[7] Vasudevan DP. Jayalakshmy S, Kumar S, et al. Assessment of pathological response of breast carcinoma in modified radical mastectomy specimens after neoadjuvant chemotherapy. Article ID 536145, International Journal of Breast Cancer 2015;2015:8.

[8] Kuerer HM, Newman LA, Smith TL, et al. Clinical course of breast cancer patients with complete pathologic primary tumour and axillary lymph node response to doxorubicin-based neoadjuvant chemotherapy. J Clin Oncol 1999;17 (2) :460-9. 
[9] Ezzat AA, Ibrahim EM, Ajarim DS, et al. Phase II study of neoadjuvant paclitaxel and cisplatin for operable and locally advanced breast cancer: analysis of 126 patients. Br J Cancer 2004;90 (5) :968-74.

[10] Elston CW, Ellis IO. Classification of malignant breast disease. In: Elston CW, Ellis IO, eds. The Breast: systemic pathology. $3^{\text {rd }}$ edn. Edinburgh, Scotland: Churchill Livingstone 1998: p. 239-47.
[11] Von Minckwitz G, Untch M, Jens-Uwe B, et al. Definition and impact of pathologic complete response on prognosis after neoadjuvant chemotherapy in various intrinsic breast cancer subtypes. J Clin Oncol 2012;30 (15) :1796-804.

[12] Galal AE, Iman I, Abdelaziz B. Response of locally advanced breast cancer to primary chemotherapy. Egyptian Journal of Surgery 2007;26

(3). 